\title{
The Informed Sampler: \\ A Discriminative Approach to Bayesian Inference in Generative Computer Vision Models
}

Varun Jampani

VARUN.JAMPANI@TUEBINGEN.MPG.DE

Max Planck Institute for Intelligent Systems, Spemannstraße 41, 72076, Tübingen, Germany

Sebastian Nowozin

SEBASTIAN.NOWOZIN@MICROSOFT.COM

Microsoft Research Cambridge, 21 Station Road, Cambridge, CB1 2FB, United Kingdom

Matthew Loper

MLOPER@TUEBINGEN.MPG.DE

Max Planck Institute for Intelligent Systems, Spemannstraße 41, 72076, Tübingen, Germany

Peter V. Gehler

PGEHLER@TUEBINGEN.MPG.DE

Max Planck Institute for Intelligent Systems, Spemannstraße 41, 72076, Tübingen, Germany

\begin{abstract}
Computer vision is hard because of a large variability in lighting, shape, and texture; in addition the image signal is non-additive due to occlusion. Generative models promised to account for this variability by accurately modelling the image formation process as a function of latent variables with prior beliefs. Bayesian posterior inference could then, in principle, explain the observation. While intuitively appealing, generative models for computer vision have largely failed to deliver on that promise due to the difficulty of posterior inference. As a result the community has favoured efficient discriminative approaches. We still believe in the usefulness of generative models in computer vision, but argue that we need to leverage existing discriminative or even heuristic computer vision methods. We implement this idea in a principled way with an informed sampler and in careful experiments demonstrate it on challenging generative models which contain renderer programs as their components. We concentrate on the problem of inverting an existing graphics rendering engine, an approach that can be understood as "Inverse Graphics". The informed sampler, using simple discriminative proposals based on existing computer vision technology, achieves significant improvements of inference.
\end{abstract}

Copyright 2015 by the author(s).

\section{Introduction}

A conceptually elegant view on computer vision is to consider a generative model of the physical image formation process. The observed image becomes a function of unobserved variables of interest (for example presence and positions of objects) and nuisance variables (for example light sources, shadows). When building such a generative model, we can think of a scene description $\theta$ that produces an image $I=G(\theta)$ using a deterministic rendering engine $G$, or more generally, results in a distribution over images, $p(I \mid \theta)$. Given an image observation $\hat{I}$ and a prior over scenes $p(\theta)$ we can then perform Bayesian inference to obtain updated beliefs $p(\theta \mid \hat{I})$. This view was advocated since the late 1970'ies (Horn, 1977, Grenander, 1976; Zhu and Mumford, 1997; Mumford and Desolneux, 2010; Mansinghka et al., 2013; Yuille and Kersten, 2006).

Now, 30 years later, we would argue that the generative approach has largely failed to deliver on its promise. The few successes of the idea have been in limited settings. In the successful examples, either the generative model was restricted to few high-level latent variables, e.g. (Oliver et al. 2000), or restricted to a set of image transformations in a fixed reference frame, e.g. (Black et al. 2000), or it modelled only a limited aspect such as object shape masks (Eslami et al. 2012), or, in the worst case, the generative model was merely used to generate training data for a discriminative model (Shotton et al., 2011). With all its intuitive appeal, its beauty and simplicity, it is fair to say that the track record of generative models in computer vision is poor. As a result, the field of computer vision is now dominated by efficient but data-hungry discriminative models, the use of empirical risk minimization for learning, and energy minimization on heuristic objective functions 


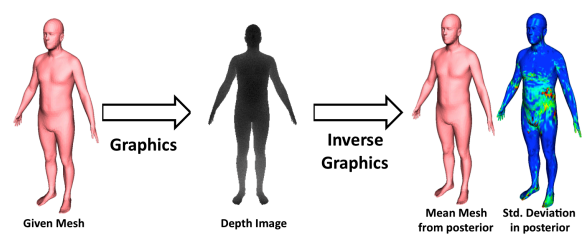

Figure 1. An example "inverse graphics" problem. A graphics engine renders a 3D body mesh and a depth image using an artificial camera. By Inverse Graphics we refer to the process of estimating the posterior probability over possible bodies given the depth image.

for inference.

Why did generative models not succeed? There are two key problems that need to be addressed, the design of an accurate generative model, and the inference therein. Modern computer graphic systems that leverage dedicated hardware setups produce a stunning level of realism with high frame rates. We believe that these systems will find its way in the design of generative models and will open up exciting modelling opportunities. This observation motivates the research question of this paper, the design of a general inference technique for efficient posterior inference in accurate computer graphics systems. As such it can be understood as an instance of Inverse Graphics (Baumgart, 1974), illustrated in Figure 1 with one of our applications.

The key problem in the generative world view is the difficulty of posterior inference at test-time. This difficulty stems from a number of reasons: first, the parameter $\theta$ is typically high-dimensional and so is the posterior. Second, given $\theta$, the image formation process realizes complex and dynamic dependency structures, for example when objects occlude or self-occlude each other. These intrinsic ambiguities result in multi-modal posterior distributions. Third, while most renderers are real-time, each simulation of the forward process is expensive and prevents exhaustive enumeration.

We believe in the usefulness of generative models for computer vision tasks, but argue that in order to overcome the substantial inference challenges we have to devise techniques that are general and allow reuse in several different models and novel scenarios. On the other hand we want to maintain correctness in terms of the probabilistic estimates that they produce. One way to improve on inference efficiency is to leverage existing computer vision features and discriminative models in order to aid inference in the generative model. In this paper, we propose the informed sampler, a Markov chain Monte Carlo (MCMC) method with discriminative proposal distributions. It can be understood as an instance of a data-driven MCMC method (Zhu et al., 2000), and our aim is to design a method that is general enough such that it can be applied across different problems and is not tailored to a particular application.

During sampling, the informed sampler leverages computer vision features and algorithms to make informed proposals for the state of latent variables and these proposals are accepted or rejected based on the generative model. The informed sampler is simple and easy to implement, but it enables inference in generative models that were out of reach for current uninformed samplers. We demonstrate this claim on challenging models that incorporate rendering engines, object occlusion, ill-posedness, and multi-modality. We carefully assess convergence statistics for the samplers to investigate their truthfulness about the probabilistic estimates. In our experiments we use existing computer vision technology: our informed sampler uses standard histogramof-gradients features (HoG) (Dalal and Triggs, 2005), and the OpenCV library, (Bradski and Kaehler, 2008), to produce informed proposals. Likewise one of our models is an existing computer vision model, the BlendSCAPE model, a parametric model of human bodies (Hirshberg et al., 2012). In Section 2, we discuss related work and explain our informed sampler approach in Section 3. Section 4 presents baseline methods and experimental setup. Then we present experimental analysis of informed sampler with three diverse problems of estimating camera extrinsics (Section 5), occlusion reasoning (Section 6) and estimating body shape (Section 7). We conclude with a discussion of future work in Section 8

\section{Related Work}

This work stands at the intersection of computer vision, computer graphics, and machine learning; it builds on previous approaches we will discuss below.

There is a vast literature on approaches to solve computer vision applications by means of generative models. We mention some works that also use an accurate graphics process as generative model. This includes applications such as indoor scene understanding (Del Pero et al. 2012), human pose estimation (Lee and Cohen 2004), hand pose estimation (de La Gorce et al. 2008) and many more. Most of these works are however interested in inferring MAP solutions, rather than the full posterior distribution.

Our method is similar in spirit to a Data Driven Markov Chain Monte Carlo (DDMCMC) methods that use a bottomup approach to help convergence of MCMC sampling. DDMCMC methods have been used in image segmentation ( $\mathrm{Tu}$ and $\mathrm{Zhu}, 2002$ ), object recognition (Zhu et al. 2000), and human pose estimation (Lee and Cohen, 2004). The idea of making Markov samplers data dependent is very general, but in the works mentioned above, lead to highly problem specific implementations, mostly using approximate likelihood functions. It is due to specialization on a 
problem domain, that the proposed samplers are not easily transferable to new problems. This is what we focus on in our work: to provide a simple, yet efficient and general inference technique for problems where an accurate forward process exists. Because our method is general we believe that it is easy to adapt to a variety of new models and tasks.

The idea to invert graphics (Baumgart, 1974) in order to understand scenes also has roots in the computer graphics community under the term "inverse rendering". The goal of inverse rendering however is to derive a direct mathematical model for the forward light transport process and then to analytically invert it. The work of (Ramamoorthi and Hanrahan, 2001) falls in this category. The authors formulate the light reflection problem as a convolution, to then understand the inverse light transport problem as a deconvolution. While this is a very elegant way to pose the problem, it does require a specification of the inverse process, a requirement generative modelling approaches try to circumvent.

Our approach can also be viewed as an instance of a probabilistic programming approach. In the recent work of (Mansinghka et al. 2013), the authors combine graphics modules in a probabilistic programming language to formulate an approximate Bayesian computation. Inference is then implemented using Metropolis-Hastings ( $\mathrm{MH})$ sampling. This approach is appealing in its generality and elegance, however we show that for our graphics problems, a plain $\mathrm{MH}$ sampling approach is not sufficient to achieve reliable inference and that our proposed informed sampler can achieve robust convergence in these challenging models. Another piece of work from (Stuhlmüller et al., 2013) is similar to our proposed inference method in that knowledge about the forward process is learned as "stochastic inverses", then applied for MCMC sampling in a Bayesian network. In the present work, we devise an MCMC sampler that we show works in both a multi-modal problem as well as for inverting an existing piece of image rendering code. In summary, our method can be understood in a similar context as the above-mentioned papers, including (Mansinghka et al. 2013).

\section{The Informed Sampler}

In general, inference about the posterior distribution is challenging because for a complex model $p(\hat{I} \mid \theta)$ no closed-form simplifications can be made. This is especially true in the case that we consider, where $p(\hat{I} \mid \theta)$ corresponds to a graphics engine rendering images. Despite this apparent complexity we observe the following: for many computer vision applications there exist well performing discriminative approaches, that, given the image, predict some parameters $\theta$ or distributions thereof. These do not correspond to the posterior distribution that we are interested in, but, intuitively the availability of discriminative inference methods should make the task of inferring $p(\theta \mid \hat{I})$ easier. Furthermore a physically accurate generative model can be used in an offline stage prior to inference to generate as many samples as we would like or can afford computationally. Again, intuitively this should allow us to prepare and summarize useful information about the distribution in order to accelerate test-time inference.

Concretely, in our case we will use a discriminative method to provide a global density $T_{G}(\hat{I})$, which we then use in a valid MCMC inference method. In the remainder of the section we first review Metropolis-Hastings Markov Chain Monte Carlo (MCMC) and then discuss our proposed informed samplers.

\subsection{Metropolis-Hastings MCMC}

The goal of any sampler is to realize independent and identically distributed samples from a given probability distribution. MCMC sampling, due to Metropolis et al. (1953) is a particular instance that generates a sequence of random variables by simulating a Markov chain. Sampling from a target distribution $\pi(\cdot)$ consists of repeating the following two steps (Liu. 2001):

1. Propose a transition using a proposal distribution $T$ and the current state $\theta_{t}$

$$
\bar{\theta} \sim T\left(\cdot \mid \theta_{t}\right)
$$

2. Accept or reject the transition based on Metropolis Hastings (MH) acceptance rule:

$$
\theta_{t+1}= \begin{cases}\bar{\theta}, & \operatorname{rand}(0,1)<\min \left(1, \frac{\pi(\bar{\theta}) T\left(\bar{\theta} \rightarrow \theta_{t}\right)}{\pi\left(\theta_{t}\right) T\left(\theta_{t} \rightarrow \bar{\theta}\right)}\right), \\ \theta_{t}, & \text { otherwise. }\end{cases}
$$

Different MCMC techniques mainly differ in the implementation of the proposal distribution $T$.

\subsection{Informed Proposal Distribution}

We use a common mixture kernel for Metropolis-Hastings sampling

$$
T_{\alpha}\left(\cdot \mid \hat{I}, \theta_{t}\right)=\alpha T_{L}\left(\cdot \mid \theta_{t}\right)+(1-\alpha) T_{G}(\cdot \mid \hat{I}) .
$$

Here $T_{L}$ is an ordinary local proposal distribution, for example a multivariate Normal distribution centered around the current sample $\theta$, and $T_{G}$ is a global proposal distribution independent of the current state. We inject knowledge by conditioning the global proposal distribution $T_{G}$ on the image observation. We learn the informed proposal $T_{G}(\cdot \mid \hat{I})$ discriminatively in an offline training stage using a nonparametric density estimator described below.

The mixture parameter $\alpha \in[0,1]$ controls the contribution of each proposal, for $\alpha=1$ we recover MH. For $\alpha=$ 


\begin{tabular}{l}
\hline Algorithm 1 Learning a global proposal $T_{G}(\theta \mid I)$ \\
\hline 1. Simulate $\left\{\left(\theta^{(i)}, I^{(i)}\right)\right\}_{i=1, \ldots, n}$ from $p(I \mid \theta) p(\theta)$ \\
2. Compute a feature representation $v\left(I^{(i)}\right)$ \\
3. Perform k-means clustering of $\left\{v\left(I^{(i)}\right)\right\}_{i}$ \\
4. For each cluster $C_{j} \subset\{1, \ldots, n\}$, fit a kernel \\
density estimate $\operatorname{KDE}\left(C_{j}\right)$ to the vectors $\theta^{\left\{C_{j}\right\}}$ \\
\hline
\end{tabular}

0 the proposal $T_{\alpha}$ would be identical to $T_{G}(\cdot \mid \hat{I})$ and the resulting Metropolis sampler would be a valid metropolized independence sampler (Liu. 2001). With $\alpha=0$ we call this baseline method Informed Independent MH (INF-INDMH). For intermediate values, $\alpha \in(0,1)$, we combine local with global moves in a valid Markov chain. We call this method Informed Metropolis Hastings (INF-MH).

\subsection{Discriminatively Learning $T_{G}$}

The key step in the construction of $T_{G}$ is to include discriminative information about the sample $\hat{I}$. Ideally we would hope to have $T_{G}$ propose global moves which improve mixing and even allow mixing between multiple modes, whereas the local proposal $T_{L}$ is responsible for exploring the density locally. To see that this is in principle possible, consider the case of a perfect global proposal, that is, $T_{G}(\cdot \mid \hat{I})=p_{\theta}(\cdot \mid \hat{I})$. In that case we would get independent samples with $\alpha=0$ because every proposal is accepted. In practice $T_{G}$ is only an approximation to $p_{\theta}(\cdot \mid \hat{I})$. If the approximation is good enough then the mixture of local and global proposals will have a high acceptance rate and explore the density rapidly.

In principle we can use any conditional density estimation technique for learning a proposal $T_{G}$ from samples. Typically high-dimensional density estimation is difficult and even more so in the conditional case; however, in our case we do have the true generating process available to provide example pairs $(\theta, I)$. Therefore we use a simple but scalable non-parametric density estimation method based on clustering a feature representation of the observed image, $v(\hat{I}) \in \mathbb{R}^{d}$. For each cluster we then estimate an unconditional density over $\theta$ using kernel density estimation (KDE). We chose this simple setup since it can easily be reused in many different scenarios, in the experiments we solve diverse problems using the same method. This method yields a valid transition kernel for which detailed balance holds.

In addition to the KDE estimate for the global transition kernel we also experimented with a random forest approach that maps the observations to transition kernels $T_{G}$. More details will be given in Section 7

For the feature representation we leverage successful discriminative features and heuristics developed in the computer vision community. Different task specific feature representations can be used in order to provide invariance

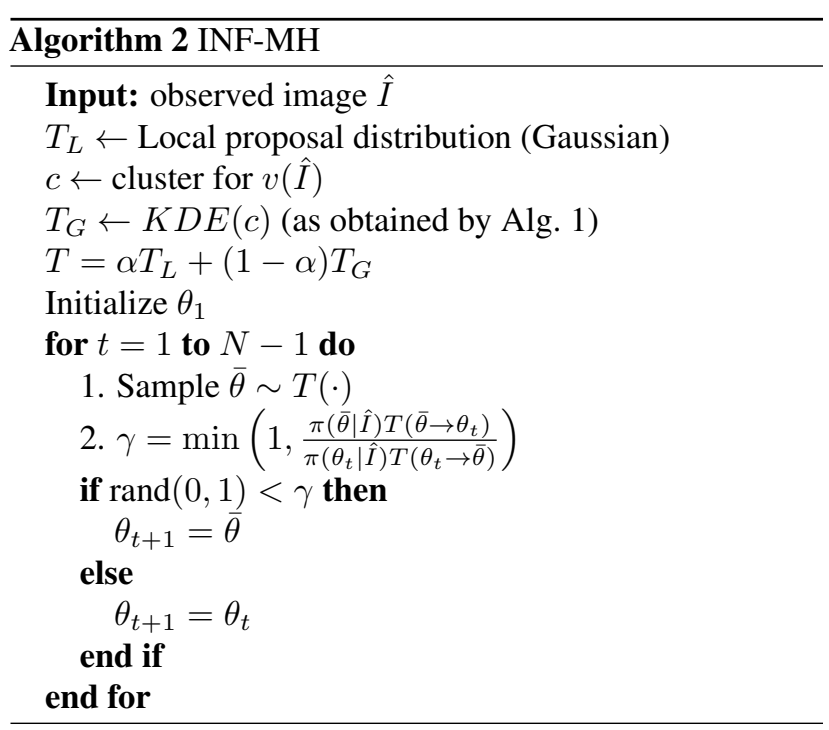

to small changes in $\theta$ and to nuisance parameters. The main inference method remains the same across problems.

We construct the KDE for each cluster and we use a relatively small kernel bandwidth in order to accurately represent the high probability regions in the posterior. This is similar in spirit to using only high probability regions as "darts" in the Darting Monte Carlo sampling technique of Sminchisescu and Welling (2011). We summarize the offline training in Algorithm 1

At test time, this method has the advantage that given an image $\hat{I}$ we only need to identify the corresponding cluster once using $v(\hat{I})$ in order to sample efficiently from the kernel density $T_{G}$. We show the full procedure in Algorithm2.

This method yields a transition kernel that is a mixture kernel of a reversible symmetric Metropolis-Hastings kernel and a metropolized independence sampler. The combined transition kernel $T$ is hence also reversible. Because the measure of each kernel dominates the support of the posterior, the kernel is ergodic and has the correct stationary distribution (Brooks et al., 2011). This ensures correctness of the inference and in the experiments we investigate the efficiency of the different methods in terms of convergence statistics.

\section{Setup and Baseline Methods}

In the remainder of the paper we demonstrate the proposed method in three different experimental setups. For all experiments, we use four parallel chains initialized at different random locations sampled from the prior. The reported numbers are median statistics over multiple test images except when noted otherwise. 


\subsection{Baseline Methods}

Metropolis Hastings (MH) Described above, corresponds to $\alpha=1$, we use a symmetric diagonal Gaussian distribution, centered at $\theta_{t}$.

Metropolis Hastings within Gibbs (MHWG) We use a Metropolis Hastings scheme in a Gibbs sampler, that is, we draw from one-dimensional conditional distributions for proposing moves and the Markov chain is updated along one dimension at a time. We further use a blocked variant of this MHWG sampler, where we update blocks of dimensions at a time, and denote it by BMHWG.

Parallel Tempering (PT) We use Parallel Tempering to address the problem of sampling from multi-modal distributions (Geyer, 1991; Swendsen and Wang, 1986). This technique is also known as "replica exchange MCMC sampling" (Hukushima and Nemoto, 1996). We run different parallel chains at different temperatures $T$, sampling $\pi(\cdot)^{\frac{1}{T}}$ and at each sampling step propose to exchange two randomly chosen chains. In our experiments we run three chains at temperature levels $T \in\{1,3,27\}$ that were found to be best working out of all combinations in $\{1,3,9,27\}$ for all experiments individually. The highest temperature levels corresponds to an almost flat distribution.

Regeneration Sampler (REG-MH) We implemented a regenerative MCMC method (Mykland et al., 1995) that performs adaption (Gilks et al., 1998) of the proposal distribution during sampling. We use the mixture kernel (Eq.11) as proposal distribution and adapt only the global part $T_{G}(\cdot \mid \hat{I})$. This is initialized as the prior over $\theta$ and at times of regeneration we fit a KDE to the already drawn samples. For comparison we used the same mixture coefficient $\alpha$ as for INF-MH (more details of this technique in A).

\subsection{MCMC Diagnostics}

We use established methods for monitoring the convergence of our MCMC method (Kass et al. 1998; Flegal et al., 2008). In particular, we report different diagnostics. We compare the different samplers with respect to the number of iterations instead of time. The forward graphics process significantly dominates the runtime and therefore the iterations in our experiments correspond linearly to the runtime.

Acceptance Rate (AR) The ratio of accepted samples to the total Markov chain length. The higher the acceptance rate, the fewer samples we need to approximate the posterior. Acceptance rate indicates how well the proposal distribution approximates the true distribution locally.

Potential Scale Reduction Factor (PSRF) The PSRF diagnostics (Gelman and Rubin, 1992, Brooks and Gelman,
1998) is derived by comparing within-chain variances with between-chain variances of sample statistics. For this, it requires independent runs of multiple chains (4 in our case) in parallel. Because our sample $\theta$ is multi-dimensional, we estimate the PSRF for each parameter dimension separately and take the maximum PSRF value as final PSRF value. A value close to one indicates that all chains characterize the same distribution. This does not imply convergence, the chains may all collectively miss a mode. However, a PSRF value much larger than one is a certain sign of lack of convergence of the chain. PSRF also indicates how well the sampler visits different modes of a multi-modal distribution.

Root Mean Square Error (RMSE) During our experiments we have access to the input parameters $\theta^{*}$ that generated the image. To assess whether the posterior distribution covers the "correct" value we report the RMSE between the posterior expectation $\mathbb{E}_{p(\cdot \mid \hat{I})}[G(\cdot)]$ and the value $G\left(\theta^{*}\right)$ of the generating input. Since there is noise being added to the observation we do not have access to the ground truth posterior expectation and therefore this measure is only an indicator. Under convergence all samplers would agree on the same correct value.

\subsection{Parameter Selection}

For each sampler we individually selected hyper-parameters that gave the best PSRF value after $10 k$ iterations. In case the PSRF does not differ for multiple values, we chose the one with highest acceptance rate. We include a detailed analysis of the baseline samplers and parameter selection in the supplementary material.

\section{Experiment: Estimating Camera Extrinsics}

We implement the following simple graphics scenario to create a challenging multi-modal problem. We render a cubical room of edge length 2 with a point light source in the center of the room $(0,0,0)$ from the viewpoint of a camera somewhere inside the room. The camera parameters are described by its $(x, y, z)$-position and the orientation, specified by yaw, pitch, and roll angles. The inference process consists of estimating the posterior over these 6D camera parameters $\theta$. See Figure 2 for two example renderings. Posterior inference is a highly multi-modal problem because the room is a cubical and thus symmetric. There are 24 different camera parameters that will result in the same image. This is also shown in Figure 2 where we plot the position and orientation (but not camera roll) of all camera parameters that create the same image. A rendering of a $200 \times 200$ image with a resolution of $32 \mathrm{bit}$ using a single core on an Intel Xeon $2.66 \mathrm{GHz}$ machine takes about $11 \mathrm{~ms}$ on average.

A small amount of isotropic Gaussian noise is added to the rendered image $G(\theta)$, using a standard deviation of $\sigma=$ 


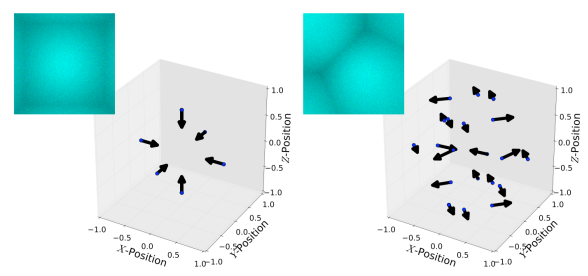

Figure 2. Two rendered room images with possible camera positions and headings that produce the same image. Not shown are the orientations; in the left example all six headings can be rolled by 90,180 , and 270 degrees for the same image.

0.02. The posterior distribution we try to infer then reads: $p(\theta \mid \hat{I}) \propto p(\hat{I} \mid \theta) p(\theta)=\mathcal{N}\left(\hat{I} \mid G(\theta), \sigma^{2}\right)$ Uniform $(\theta)$. The uniform prior over location parameters ranges between -1.0 and 1.0 and the prior over angle parameters is modelled with wrapped uniform distribution over $[-\pi, \pi]$.

To learn the informed part of the proposal distribution from data, we computed a histogram of oriented gradients (HOG) descriptor (Dalal and Triggs, 2005) from the image, using 9 gradient orientations and cells of size $20 \times 20$ yielding a feature vector $v(I) \in \mathbb{R}^{900}$. We generated $300 k$ training images using a uniform prior over the camera extrinsic parameters, and performed $\mathrm{k}$-means using $5 \mathrm{k}$ cluster centers based on the HOG feature vector. For each cluster cell, we then computed and stored a KDE for the 6 dimensional camera parameters, following the steps in Algorithm 1 . As test data, we create 30 images using extrinsic parameters sampled uniform at random over their range.

\subsection{Results}

We show results in Figure 3. We observe that both $\mathrm{MH}$ and PT yield low acceptance rate compared to other methods. However parallel tempering appears to overcome the multi-modality better and improves over $\mathrm{MH}$ in terms of convergence. The same holds for the regeneration technique, we observe many regenerations, good convergence and AR. Both INF-INDMH and INF-MH converge quickly.

In this experimental setup have access to the different exact modes, there are 24 different ones. We analyze how quickly the samplers visit the modes and whether or not they capture all of them. For ever different instance the pairwise distances between the modes changes, therefore we chose to define "visiting a mode" in the following way. We compute a Voronoi tesselation with the modes as centers. A mode is visited if a sample falls into its corresponding Voronoi cell, that is, it is closer than to any other mode. Sampling uniform at random would quickly find the modes (depending on the cell sizes) but is not a valid sampler. We also experimented with balls of different radii around the modes and found a similar behaviour to the one we report here. Figure 3 (right) shows results for various samplers. We find that INF-MH discovers different modes quicker when compared to other baseline samplers. Just sampling from the global proposal distribution INF-INDMH is initially visiting more modes (it is not being held back by local steps) but is dominated by INF-MH over some range. This indicates that the mixture kernel takes advantage of both local and global moves, either one of them is exploring slower. Also in most examples all samplers miss some modes under our definition, the average number of discovered modes is 21 for INF-MH and even lower for MH.

Figure 14(c) shows the effect of mixture coefficient $(\alpha)$ on the informed sampling INF-MH. Since there is no significant difference in PSRF values for $0 \leq \alpha \leq 0.7$, we chose 0.7 due to its high acceptance rate. Likewise, the parameters of the baseline samplers are chosen based on the PSRF and acceptance rate metrics. See supplementary material for the analysis of the baseline samplers and the parameter selection.
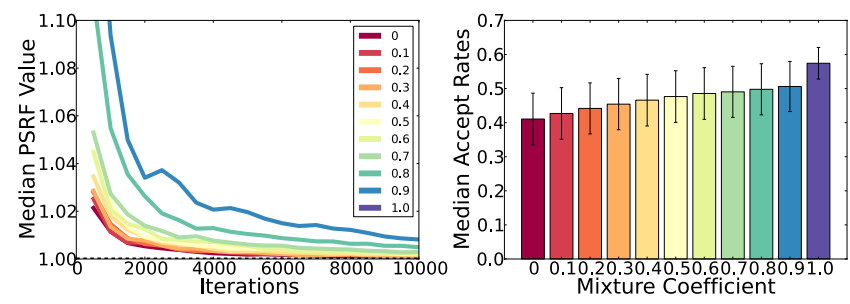

Figure 4. Role of mixture coefficient. PRSFs and Acceptance rates corresponding to various mixture coefficients $(\alpha)$ of INF-MH sampling in 'Estimating Camera Extrinsics' experiment.

We also tested the MHWG sampler and found that it did not converge even after $100 k$ iterations, with a PSRF value around 3 . This is to be expected since single variable updates will not traverse the multi-modal posterior distributions fast enough due to the high correlation of the camera parameters. In Figure 5 we plot the median auto-correlation of samples obtained by different sampling techniques, separately for each of the six extrinsic camera parameters. The informed sampling approach (INF-MH and INF-INDMH) appears to produce samples which are more independent compared to other baseline samplers.

As expected, some knowledge of the multi-modal structure of the posterior needs to be available for the sampler to perform well. The methods INF-INDMH and INF-MH have this information and perform better than baseline methods and REG-MH.

\section{Experiment: Occluding Tiles}

In a second experiment we render images depicting a fixed number of six quadratic tiles placed at a random location $x, y$ in the image at a random depth $z$ and orientation $\theta$. 

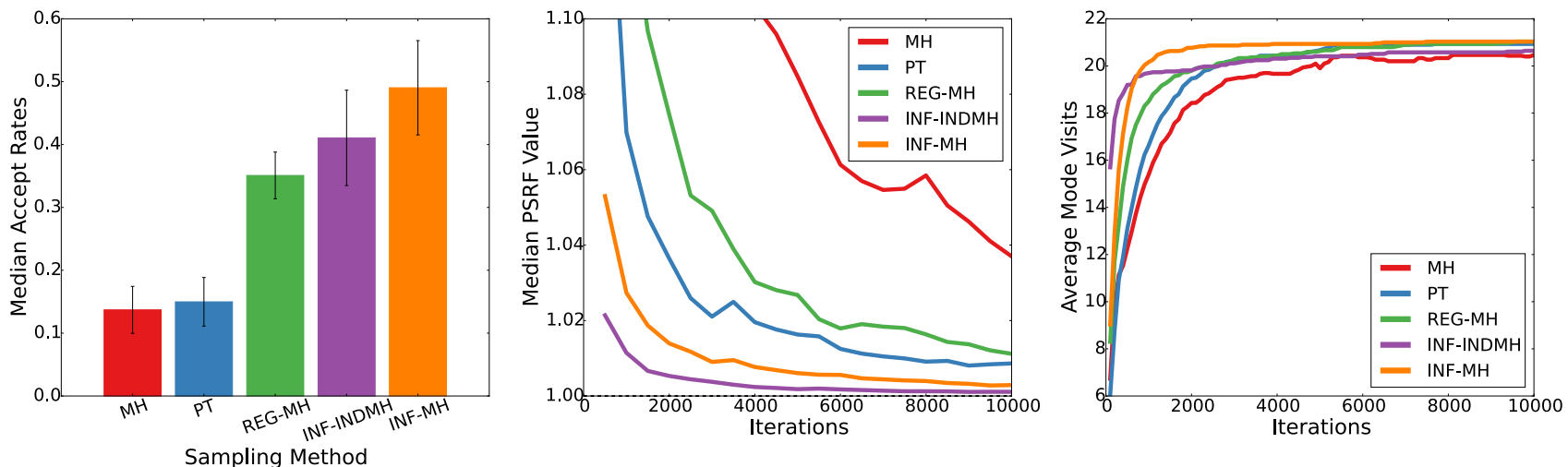

Figure 3. Results of the 'Estimating Camera Extrinsics' experiment. Acceptance Rates (left), PSRFs (middle), and Average number of modes visited (right) for different sampling methods. We plot the median/average statistics over 30 test examples.
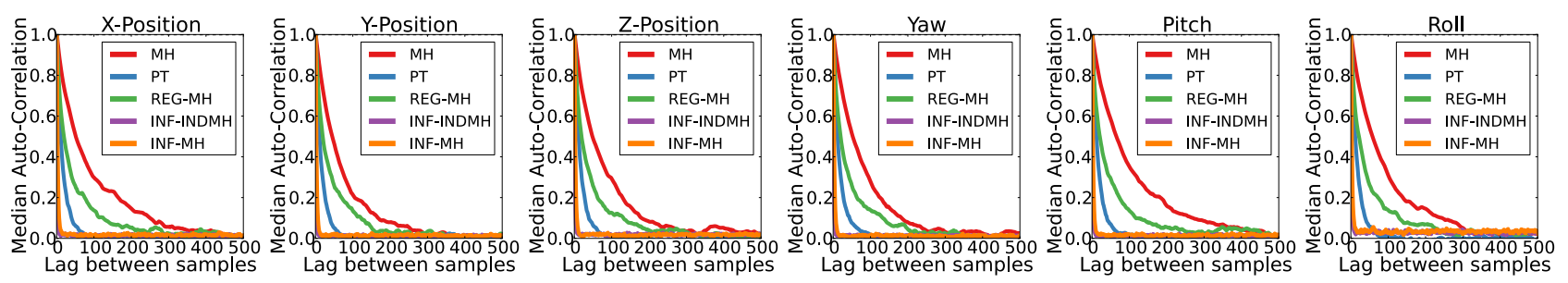

Figure 5. Auto-Correlation of samples obtained by different sampling techniques in camera extrinsics experiment, for each of the six extrinsic camera parameters.

We blur the image and add a bit of Gaussian random noise $(\sigma=0.02)$. An example is depicted in Figure 6 a a), note that all the tiles are of the same size, but farther away tiles look smaller. A rendering of one $200 \times 200$ image takes about $25 \mathrm{~ms}$ on average. Here, as prior, we again use the uniform distribution over the 3D cube for tile location parameters, and wrapped uniform distribution over $\left[-\frac{\pi}{4}, \frac{\pi}{4}\right]$ for tile orientation angle. To avoid label switching issues, each tile is given a fixed color and is not changed during the inference.

We chose this experiment such that it resembles the "dead leaves model" of Lee et al. (2001), because it has properties that are commonplace in computer vision. It is a scene composed of several objects that are independent, except for occlusion, which complicates the problem. If occlusion did not exist, the task is readily solved using a standard OpenCV (Bradski and Kaehler, 2008) rectangle finding algorithm (minAreaRect). The output of such an algorithm can be seen in Figure 6(c), and we use this algorithm as a discriminative source of information. This problem is higher dimensional than the previous one (24, due to 6 tiles of 4 parameters). Inference becomes more challenging in higher dimension and our approach without modification does not scale well with increasing dimensionality. One way to approach this problem, is to factorize the joint distribution into blocks and learn informed proposals separately. In the present experiment, we observed that both baseline samplers and the plain informed sampling fail when proposing all parameters jointly. Since the tiles are independent except for the occlusion, we can approximate the full joint distribution as product of block distributions where each block corresponds to the parameters of a single tile. To estimate the full posterior distribution, we learn global proposal distributions for each block separately and use a block-Gibbs like scheme in our sampler where we propose changes to one tile at a time, alternating between tiles.

The experimental protocol is the same as before, we render $500 k$ images, apply the OpenCV algorithm to fit rectangles and take their found four parameters as features for clustering (10k clusters). Again KDE distributions are fit to each cluster and at test time, we assign the observed image to its corresponding cluster. The KDE in that chosen cluster determines the global sampler $T_{G}$ for that tile. We then use $T_{G}$ to propose an update to all 4 parameters of the tile. We refer to this procedure as INF-BMHWG. Empirically we find $\alpha=0.8$ to be optimal for INF-BMHWG sampling.

\subsection{Results}

An example result is shown in Figure 6. We found that the the MH and INF-MH samplers fail entirely on this problem. Both use a proposal distribution for the entire state and due to the high dimensions there is almost no acceptance $(<$ 


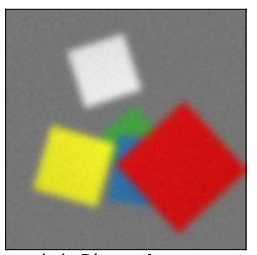

(a) Given Image

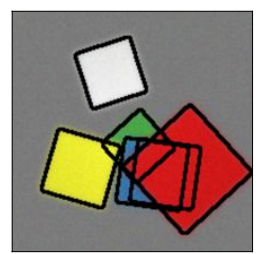

(b) Ground Truth

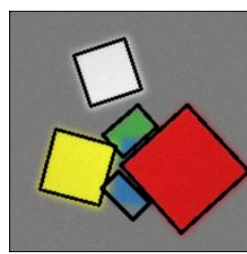

(c) OpenCV Heuristic

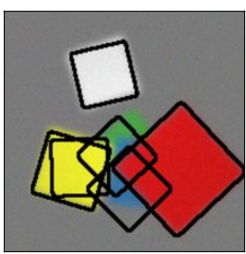

(d) MHWG

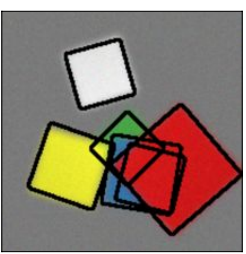

(e) INF-BMHWG

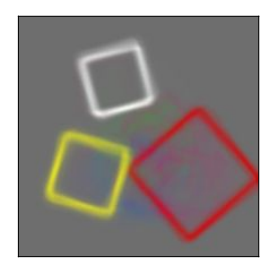

(f) INF-BMHWG (Mean)

Figure 6. A visual result in 'Occluding Tiles' experiment. (a) A sample rendered image, (b) Ground truth squares, and most probable estimates from 5000 samples obtained by (c) MHWG sampler (best baseline) and (d) the INF-BMHWG sampler. (f) Posterior expectation of the square boundaries obtained by INF-BMHWG sampling. (The first 2000 samples are discarded as burn-in)

$1 \%$ ) and thus they do not reach convergence. The MHWG sampler, updating one dimension at a time, is found to be the best among the baseline samplers with acceptance rate of around $42 \%$, followed by a block sampler that samples each tile separately. The OpenCV algorithm produces a reasonable initial guess but fails in occlusion cases.

The block wise informed sampler INF-BMHWG converges quicker, with higher acceptance rates $(\approx 53 \%)$, and lower reconstruction error. The median curves for 10 test examples are shown in Figure 7. INF-BMHWG by far produces lower reconstruction errors. Also in Fig 6(f) the posterior distribution is visualized, fully visible tiles are more localized, position and orientation of occluded tiles more uncertain. Figure 12 in the appendix shows some more visual results. Although the model is relatively simple, all the baseline samplers perform poorly and discriminative information is crucial to enable accurate inference. Here the discriminative information is provided by a readily available heuristic in the OpenCV library.

This experiment illustrates a variation of the informed sampling strategy that can be applied to sampling from highdimensional distributions. Inference methods for general high-dimensional distributions is an active area of research and intrinsically difficult. The occluding tiles experiment is simple but illustrates this point, namely that all non-block baseline samplers fail. Block sampling is a common strategy in such scenarios and many computer vision problems have such block-structure. Again the informed sampler improves in convergence speed over the baseline method. Other techniques that produce better fits to the conditional (block-)marginals should give faster convergence.

\section{Experiment: Estimating Body Shape}

The last experiment is motivated by a real world problem: estimating the 3D body shape of a person from a single static depth image. With the recent availability of cheap active depth sensors, the use of RGBD data has become ubiquitous in computer vision (Shao et al., 2013, Jungong et al. 2013).

To represent a human body we use the BlendSCAPE model (Hirshberg et al., 2012), which updates the originally proposed SCAPE model (Anguelov et al. 2005) with better training and blend weights. This model produces a 3D mesh of a human body as shown in Figure 8 as a function of shape and pose parameters. The shape parameters allow us to represent bodies of many builds and sizes, and includes a statistical characterization (being roughly Gaussian). These parameters control directions in deformation space, which were learned from a corpus of roughly 2000 3D mesh models registered to scans of human bodies via PCA. The pose parameters are joint angles which indirectly control local orientations of predefined parts of the model.

Our model uses 57 pose parameters and any number of shape parameters to produce a 3D mesh with 10,777 vertices. We use the first 7 SCAPE components to represent the shape of a person. The camera viewpoint, orientation, and pose of the person is held fixed. Thus a rendering process takes $\theta \in$ $\mathbb{R}^{7}$, generates a 3D mesh representation of it and projects it through a virtual depth camera to create a depth image of the person. This can be done in various resolutions, we chose $430 \times 260$ with depth values represented as 32 bit numbers in the interval $[0,4]$. On average, a full render path takes about $28 \mathrm{~ms}$. We add Gaussian noise with standard deviation of 0.02 to the created depth image. See Fig 8 (left) for an example.

We used very simple low level features for feature representation. In order to learn the global proposal distribution we compute depth histogram features on a $15 \times 10$ grid on the image. For each cell we record the mean and variance of the depth values. Additionally we add the height and the width of the body silhouette as features resulting in a feature vector $v(I) \in \mathbb{R}^{302}$. As normalization, each feature dimension is divided by the maximum value in the training set. We used $400 k$ training images sampled from the standard normal prior distribution and $10 k$ clusters to learn the KDE proposal distributions in each cluster cell.

For this experiment we also experimented with a different conditional density estimation approach using a forest of random regression trees (Breiman et al., 1984, Breiman, 2001). In the previous experiments, utilizing the KDE estimates, the discriminative information entered through the 

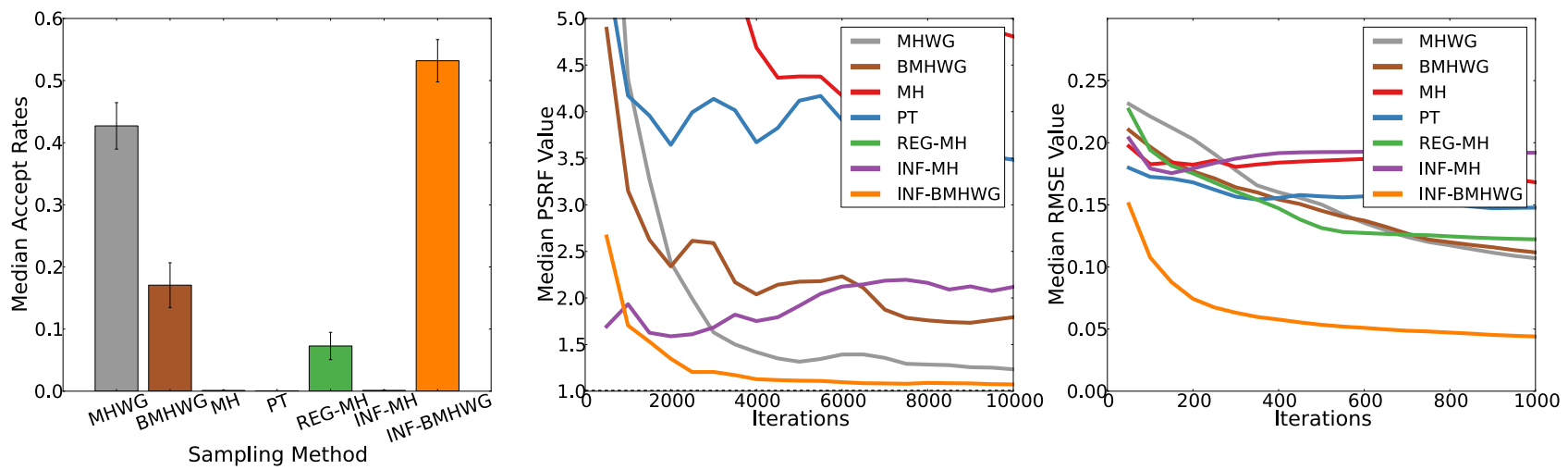

Figure 7. Results of the 'Occluding Tiles' experiment. Acceptance Rates (left), PSRFs (middle), and RMSEs (right) for different sampling methods. Median results for 10 test examples.

feature representation. Then, suppose if there was no relation between some observed features and the variables that we are trying to infer, we would require a large number of samples to reliably estimate the densities in the different clusters. The regression forest can adaptively partition the parameter space based on observed features and is able to ignore uninformative features, thus may lead to better fits of the conditional densities. It can thus be understood as the adaptive version of the $\mathrm{k}$-Means clustering technique that solely relies on the used metric (Euclidean in our case).

In particular, we use the same features as for k-means clustering but grow the regression trees using a mean square error criterion for scoring the split functions. A forest of 10 binary trees with a depth of 15 is grown, with the constraint of having a minimum of 40 training points per leaf node. Then for each of the leaf nodes, a KDE is trained as before. At test time the regression forest yields a mixture of KDEs as the global proposal distribution. We denote this method as INF-RFMH in the experiments.

Instead of placing using one KDE model for each cluster, we could also explore a regression approach, for example using a discriminative linear regression model to map observations into proposal distributions. By using informative covariates in the regression model one should be able to overcome the curse of dimensionality. Such a semi-parametric approach would allow to capture explicit parametric dependencies of the variables (for example linear dependencies) and combine them with non-parametric estimates of the residuals. We are exploring this technique as future work.

Again, we chose parameters for all samplers individually, based on empirical mixing rates. For informed samplers, we chose $\alpha=0.8$, and a local proposal standard deviation of 0.05 . The full analysis for all samplers is included in the supplementary material.

\subsection{Results}

We tested the different approaches on 10 test images that are generated by parameters drawn from the standard normal prior distribution. Figure 9 summarizes the results of the sampling methods. We make the following observations. The baselines methods MH, MHWG, and PT show inferior convergence results and $\mathrm{MH}$ and $\mathrm{PT}$ also suffer from lower acceptance rates. Just sampling from the distribution of the discriminative step (INF-INDMH) is not enough, because the low acceptance rate indicates that the global proposals do not represent the correct posterior distribution. However, combined with a local proposal in a mixture kernel, we achieve a higher acceptance rate, faster convergence and a decrease in RMSE. The regression forest approach has slower convergence than INF-MH. In this example, the regeneration sampler REG-MH does not improve over simpler baseline methods. We attribute this to rare regenerations which may be improved with more specialized methods.

We believe that our simple choice of depth image representation can also significantly be improved on. For example, features can be computed from identified body parts, something that the simple histogram features have not taken into account. In the computer vision literature some discriminative approaches for pose estimation do exist, most prominent being the influential work on pose recovery in parts for the Kinect XBox system (Shotton et al., 2011). In future work we plan to use similar methods to deal with pose variation and complicated dependencies between parameters and observations.

\subsection{D Mesh Reconstruction}

In Figure 8 we show a sample 3D body mesh reconstruction result using the INF-MH sampler after only 1000 iterations. We visualized the difference of the mean posterior and the ground truth 3D mesh in terms of mesh edge directions. One 

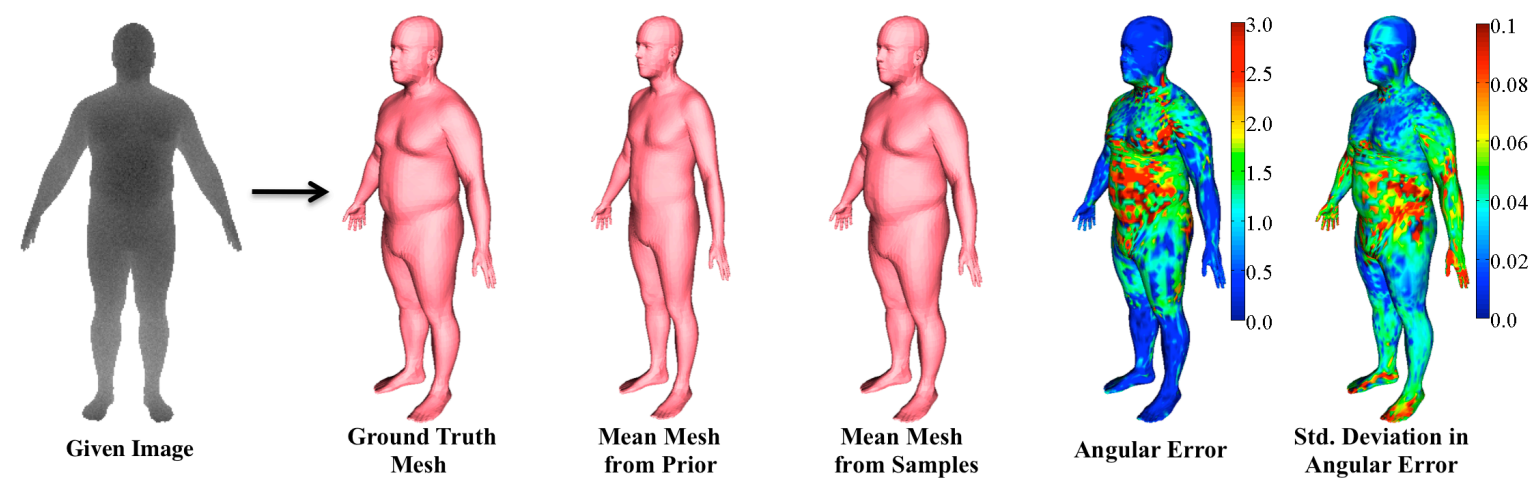

Figure 8. Inference of body shape from a depth image. A sample test result showing the result of 3D mesh reconstruction with the first 1000 samples obtained using our INF-MH sampling method. We visualize the angular error (in degrees) between the estimated and ground truth edge and project onto the mesh.
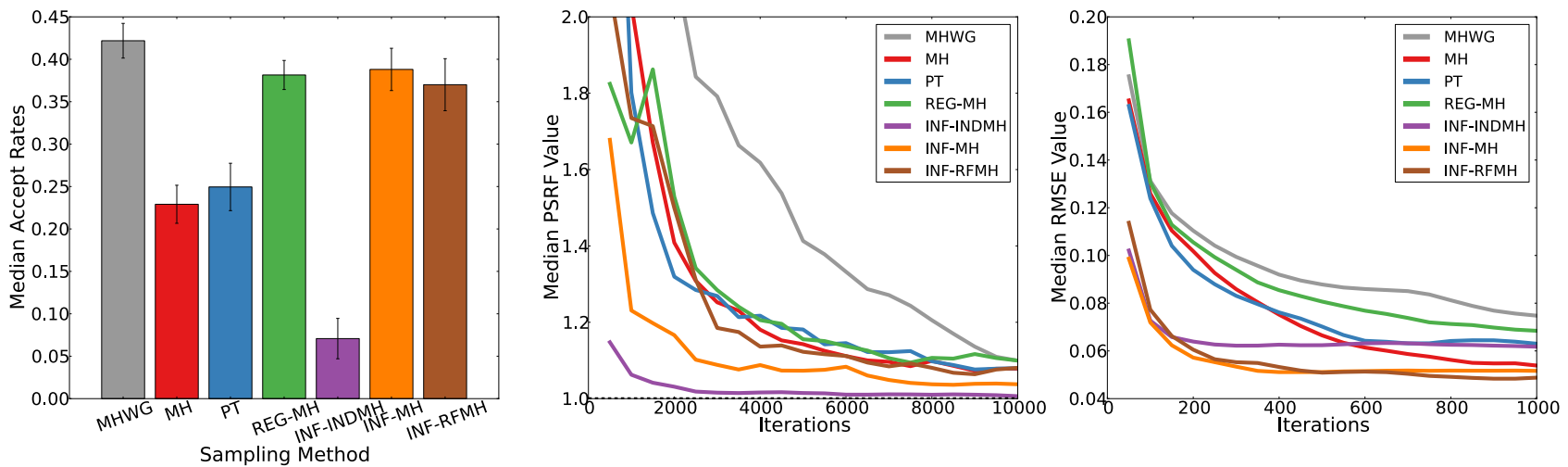

Figure 9. Results of the 'Body Shape' experiment. Acceptance Rates (left), PSRFs (middle), and RMSEs (right) for different sampling methods in the body shape experiment. Median results over 10 test examples.

can observe that most differences are in the belly region and the feet of the person. The retrieved posterior distribution allows us to assess the model uncertainty. To visualize the posterior variance we record standard deviation over the edge directions for all mesh edges. This is backprojected to achieve the visualization in Figure 8 (right). We see that posterior variance is higher in regions of higher error, that is, our model predicts its own uncertainty correctly (Dawid, 1982). In a real-world body scanning scenario, this information will be beneficial; for example, when scanning from multiple viewpoints or in an experimental design scenario, it helps in selecting the next best pose and viewpoint to record. Figure 13 shows more 3D mesh reconstruction results using our sampling approach.

\subsection{Body Measurements}

Predicting body measurements has many applications including clothing, sizing and ergonomic design. Given pixel observations, one may wish to infer a distribution over mea- surements (such as height and chest circumference). Fortunately, our original shape training corpus includes a host of 47 different per-subject measurements, obtained by professional anthropometrists; this allows us to relate shape parameters to measurements. Among many possible forms of regression, regularized linear regression (Zou and Hastie. 2005) was found to best predict measurements from shape parameters. This linear relationship allows us to transform any posterior distribution over SCAPE parameters into a posterior over measurements, as shown in Figure 10 We report for three randomly chosen subjects' (S1, S2, and S3) results on three out of the 47 measurements. The dashed lines corresponds to ground truth values. Our estimate not only faithfully recovers the true value but also yields a characterization of the full conditional posterior.

\subsection{Incomplete Evidence}

Another advantage of using a generative model is the ability to reason with missing observations. We perform a simple 


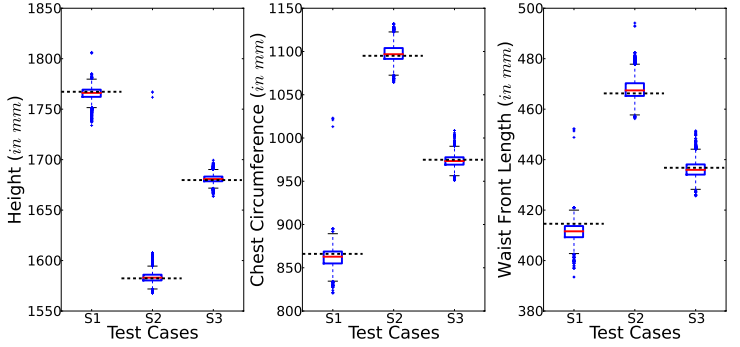

Figure 10. Body measurements with quantified uncertainty. Box plots of three body measurements for three test subjects, computed from the first $10 k$ samples obtained by the INF-MH sampler. Dotted lines indicate measurements corresponding to ground truth SCAPE parameters.

experiment by occluding a portion of the observed depth image. We use the same inference and learning codes, with the same parametrization and features as in the non-occlusion case but retrain the model to account for the changes in the forward process. The result of INF-MH, computed on the first $10 k$ samples is shown in Fig. 11 . The $3 \mathrm{D}$ reconstruction is reasonable even under large occlusion; the error and the edge direction variance did increase as expected.

\section{Discussion and Conclusions}

This work proposes a method to incorporate discriminative methods into Bayesian inference in a principled way. We augment a sampling technique with discriminative information to enable inference with global accurate generative models. Empirical results on three challenging and diverse computer vision experiments are discussed. We carefully analyse the convergence behaviour of several different baselines and find that the informed sampler performs well across all different scenarios. This sampler is applicable to general scenarios and in this work we leverage the accurate forward process for offline training, a setting frequently found in computer vision applications. The main focus is the generality of the approach, this inference technique should be applicable to many different problems and not be tailored to a particular problem.

We show that even for very simple scenarios, most baseline samplers perform poorly or fail completely. By including a global image-conditioned proposal distribution that is informed through discriminative inference we can improve sampling performance. We deliberately use a simple learning technique (KDEs on k-Means cluster cells and a forest of regression trees) to enable easy reuse in other applications. Using stronger and more tailored discriminative models should lead to better performance. We see this as a way where top-down inference is combined with bottom-up proposals in a probabilistic setting.

There are some avenues for future work; we understand

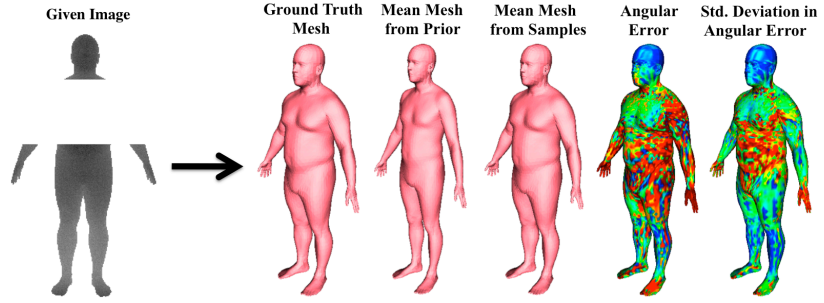

Figure 11. Inference with incomplete evidence. Mean 3D mesh and corresponding errors and uncertainties (std. deviations) in mesh edge directions, for the same test case as in figure 8 , computed from first $10 k$ samples of our INF-MH sampling method with (bottom row) occlusion mask in image evidence. (blue indicates small values and red indicates high values)

this method as an initial step into the direction of general inference techniques for accurate generative computer vision models. Identifying conditional dependence structure should improve results, e.g. recently Stuhlmüller et al. (2013) used structure in Bayesian networks to identify such dependencies. One assumption in our work is that we use an accurate generative model. Relaxing this assumption to allow for more general scenarios where the generative model is known only approximately is important future work. In particular for high-level computer vision problems such as scene or object understanding there are no accurate generative models available yet but there is a clear trend towards physically more accurate 3D representations of the world. This more general setting is different to the one we consider in this paper, but we believe that some ideas can be carried over. For example, we could create the informed proposal distributions from manually annotated data that is readily available in many computer vision data sets. Another problem domain are trans-dimensional models, that require different sampling techniques like reversible jump MCMC methods (Green, 1995, Brooks et al., 2011). We are investigating general techniques to "inform" this sampler in similar ways as described in this manuscript.

We believe that generative models are useful in many computer vision scenarios and that the interplay between computer graphics and computer vision is a prime candidate for studying probabilistic inference and probabilistic programming (Mansinghka et al., 2013). However, current inference techniques need to be improved on many fronts: efficiency, ease of usability, and generality. Our method is a step towards this direction: the informed sampler leverages the power of existing discriminative and heuristic techniques to enable a principled Bayesian treatment in rich generative models. Our emphasis is on generality; we aimed to create a method that can be easily reused in other scenarios with existing code bases. The presented results are a successful example of the inversion of an involved rendering pass. In the future we plan to investigate ways to combine existing computer vision techniques with principled generative 
models, with the aim of being general rather than problem specific.

\section{Appendix}

\section{A. Regeneration Sampler (REG-MH)}

Adapting the proposal distribution with existing MCMC samples is not straight-forward as this would potentially violate the Markov property of the chain (Atchadé and Rosenthal 2005). One approach is to identify times of regeneration at which the chain can be restarted and the proposal distribution can be adapted using samples drawn previously. Several approaches to identify good regeneration times in a general Markov chain have been proposed (Athreya and Ney, 1978, Nummelin, 1978). We build on (Mykland et al. $1995)$ that proposed two splitting methods for finding the regeneration times. Here, we briefly describe the method that we implemented in this study.

Let the present state of the sampler be $x$ and let the independent global proposal distribution be $T_{G}$. When $y \sim T_{G}$ is accepted according to the $\mathrm{MH}$ acceptance rule, the probability of a regeneration is given by:

$r(x, y)= \begin{cases}\max \left\{\frac{c}{w(x)}, \frac{c}{w(y)}\right\}, & \text { if } w(x)>c \text { and } w(y)>c, \\ \max \left\{\frac{w(x)}{c}, \frac{w(y)}{c}\right\}, & \text { if } w(x)<c \text { and } w(y)<c, \\ 1, & \text { otherwise, }\end{cases}$

where $c>0$ is an arbitrary constant and $w(x)=\frac{\pi(x)}{T_{G}(x)}$. The value of $c$ can be set to maximize the regeneration probability. At every sampling step, if a sample from the independent proposal distribution is accepted, we compute regeneration probability using equation 2 . If a regeneration occurs, the present sample is discarded and replaced with one from the independent proposal distribution $T_{G}$. We use the same mixture proposal distribution as in our informed sampling approach where we initialize the global proposal $T_{G}$ with a prior distribution and at times of regeneration fit a KDE to the existing samples. This becomes the new adapted distribution $T_{G}$. Refer to (Mykland et al., 1995) for more details of this regeneration technique. In the work of (Ahn et al. 2013) this regeneration technique is used with success in a Darting Monte Carlo sampler.

\section{B. Additional Qualitative Results}

\section{B.1. Occluding Tiles}

In Figure 12 more qualitative results of the occluding tiles experiment are shown. The informed sampling approach (INF-BMHWG) is better than the best baseline (MHWG). This still is a very challenging problem since the parameters for occluded tiles are flat over a large region. Some of the posterior variance of the occluded tiles is already captured by the informed sampler.

\section{B.2. Body Shape}

Figure 13 shows some more results of 3D mesh reconstruction using posterior samples obtained by our informed sampling INF-MH.

\section{References}

S. Ahn, Y. Chen, and M. Welling. Distributed and adaptive darting Monte Carlo through regenerations. In Proceedings of the 16th International Conference on Artificial Intelligence and Statistics (AI Stats), 2013.

D. Anguelov, P. Srinivasan, D. Koller, S. Thrun, J. Rodgers, and J. Davis. Scape: shape completion and animation of people. In ACM Transactions on Graphics (TOG), volume 24, pages 408-416. ACM, 2005.

Y. F. Atchadé and J. S. Rosenthal. On adaptive Markov chain Monte Carlo algorithms. Bernoulli, 11(5):815-828, 2005.

K. B. Athreya and P. Ney. A new approach to the limit theory of recurrent Markov chains. Transactions of the American Mathematical Society, 245:493-501, 1978.

B. G. Baumgart. Geometric Modeling for Computer Vision. $\mathrm{PhD}$ thesis, Stanford university, 1974.

M. J. Black, D. J. Fleet, and Y. Yacoob. Robustly estimating changes in image appearance. Computer Vision and Image Understanding, 78(1):8-31, 2000.

G. Bradski and A. Kaehler. Learning OpenCV: Computer vision with the OpenCV library. OReilly, 2008.

L. Breiman. Random forests. Machine Learning, 45(1): 5-32, 2001.

L. Breiman, J. H. Friedman, R. A. Olshen, and C. J. Stone. Classification and Regression Trees. Wadsworth, Belmont, 1984.

S. Brooks and A. Gelman. General methods for monitoring convergence of iterative simulations. Journal of Computational and Graphical Statistics, 7:434-455, 1998.

S. Brooks, A. Gelman, G. Jones, and X.-L. Meng. Handbook of Markov Chain Monte Carlo. CRC Press, 2011.

N. Dalal and B. Triggs. Histograms of oriented gradients for human detection. In Computer Vision and Pattern Recognition, volume 1, pages 886-893, 2005.

P. A. Dawid. The well-calibrated Bayesian. Journal of the American Statistical Association, 77(379):605-610, 1982. 
M. de La Gorce, N. Paragios, and D. J. Fleet. Model-based hand tracking with texture, shading and self-occlusions. In Computer Vision and Pattern Recognition, 2008.

L. Del Pero, J. Bowdish, D. Fried, B. Kermgard, E. Hartley, and K. Barnard. Bayesian geometric modeling of indoor scenes. In Computer Vision and Pattern Recognition, 2012.

S. M. A. Eslami, N. Heess, and J. M. Winn. The shape Boltzmann machine: A strong model of object shape. In Computer Vision and Pattern Recognition, pages 406413. IEEE, 2012.

J. M. Flegal, M. Haran, and G. L. Jones. Markov chain Monte Carlo: Can we trust the third significant figure? Statistical Science, 23(2):250-260, 2008.

A. Gelman and D. Rubin. Inference from iterative simulation using multiple sequences. Statistical Science, 7: 457-511, 1992.

C. J. Geyer. Markov chain Monte Carlo maximum likelihood. In Proceedings of the 23rd Symposium on the Interface, pages 156-163, 1991.

W. R. Gilks, G. O. Roberts, and S. K. Sahu. Adaptive Markov chain Monte Carlo through regeneration. Journal of the American statistical association, 93(443):10451054, 1998.

P. J. Green. Reversible jump Markov chain Monte Carlo computation and Bayesian model determination. Biometrika, 82(4):711-732, 1995.

U. Grenander. Pattern Synthesis - Lectures in Pattern Theory. Springer, New York, 1976.

D. A. Hirshberg, M. Loper, E. Rachlin, and M. J. Black. Coregistration: simultaneous alignment and modeling of articulated 3d shape. In European conference on Computer Vision, pages 242-255, 2012.

B. K. P. Horn. Understanding image intensities. Artificial Intelligence, 8:201-231, 1977.

K. Hukushima and K. Nemoto. Exchange Monte Carlo method and application to spin glass simulations. Journal of the Physical Society of Japan, 65(6):1604-1608, 1996.

H. Jungong, S. Ling, X. Dong, and J. Shotton. Enhanced computer vision with Microsoft Kinect sensor: A review. IEEE Transactions on Cybernetics, 43(5):13181334, Oct 2013.

R. E. Kass, B. P. Carlin, A. Gelman, and R. M. Neal. Markov chain Monte Carlo in practice: A roundtable discussion. The American Statistician, 52:93-100, 1998.
A. B. Lee, D. Mumford, and J. Huang. Occlusion models for natural images: A statistical study of a scale-invariant dead leaves model. International Journal of Computer Vision, 41(1-2):35-59, 2001.

M. W. Lee and I. Cohen. Proposal maps driven MCMC for estimating human body pose in static images. In Computer Vision and Pattern Recognition, 2004.

J. S. Liu. Monte Carlo Strategies in Scientific Computing. Springer Series in Statistics. New York, 2001.

V. Mansinghka, T. D. Kulkarni, Y. N. Perov, and J. Tenenbaum. Approximate Bayesian image interpretation using generative probabilistic graphics programs. In Advances in Neural Information Processing Systems, pages 1520 1528, 2013.

N. Metropolis, A. W. Rosenbluth, M. N. Rosenbluth, A. H. Teller, and E. Teller. Equation of state calculations by fast computing machines. The journal of chemical physics, 21:1087, 1953.

D. Mumford and A. Desolneux. Pattern Theory: The Stochastic Analysis of Real-World Signals. 2010.

P. Mykland, L. Tierney, and B. Yu. Regeneration in Markov chain samplers. Journal of the American Statistical Association, 90(429):233-241, 1995.

E. Nummelin. A splitting technique for Harris recurrent Markov chains. Zeitschrift für Wahrscheinlichkeitstheorie und verwandte Gebiete, 43(4):309-318, 1978.

N. Oliver, B. Rosario, and A. Pentland. A Bayesian computer vision system for modeling human interactions. IEEE Trans. Pattern Anal. Mach. Intell, 22(8):831-843, 2000.

R. Ramamoorthi and P. Hanrahan. A signal-processing framework for inverse rendering. In Computer graphics and interactive techniques, pages 117-128. ACM, 2001.

L. Shao, J. Han, D. Xu, and J. Shotton. Computer vision for RGB-D sensors: Kinect and its applications. IEEE Transactions on Cybernetics, 43(5):1314-1317, 2013.

J. Shotton, A. Fitzgibbon, M. Cook, T. Sharp, M. Finocchio, R. Moore, A. Kipman, and A. Blake. Real-time human pose recognition in parts from single depth images. In Computer Vision and Pattern Recognition, 2011.

C. Sminchisescu and M. Welling. Generalized darting Monte Carlo. Pattern Recognition, 44(10):2738-2748, 2011.

A. Stuhlmüller, J. Taylor, and N. Goodman. Learning stochastic inverses. In Advances in Neural Information Processing Systems, pages 3048-3056, 2013. 
R. H. Swendsen and J.-S. Wang. Replica Monte Carlo simulation of spin-glasses. Physical Review Letters, 57 (21):2607, 1986.

Z. Tu and S.-C. Zhu. Image segmentation by data-driven Markov chain Monte Carlo. Pattern Analysis and Machine Intelligence, IEEE Transactions on, 24(5):657-673, 2002.

A. Yuille and D. Kersten. Vision as Bayesian inference: analysis by synthesis? Trends in cognitive sciences, 10 (7):301-308, 2006.

S. C. Zhu and D. Mumford. Learning generic prior models for visual computation. In Computer Vision and Pattern Recognition, pages 463-469. IEEE, 1997.

S.-C. Zhu, R. Zhang, and Z. Tu. Integrating bottom-up/topdown for object recognition by data driven Markov chain Monte Carlo. In Computer Vision and Pattern Recognition, volume 1, pages 738-745, 2000.

H. Zou and T. Hastie. Regularization and variable selection via the elastic net. Journal of the Royal Statistical Society: Series B (Statistical Methodology), 67(2):301-320, 2005. 

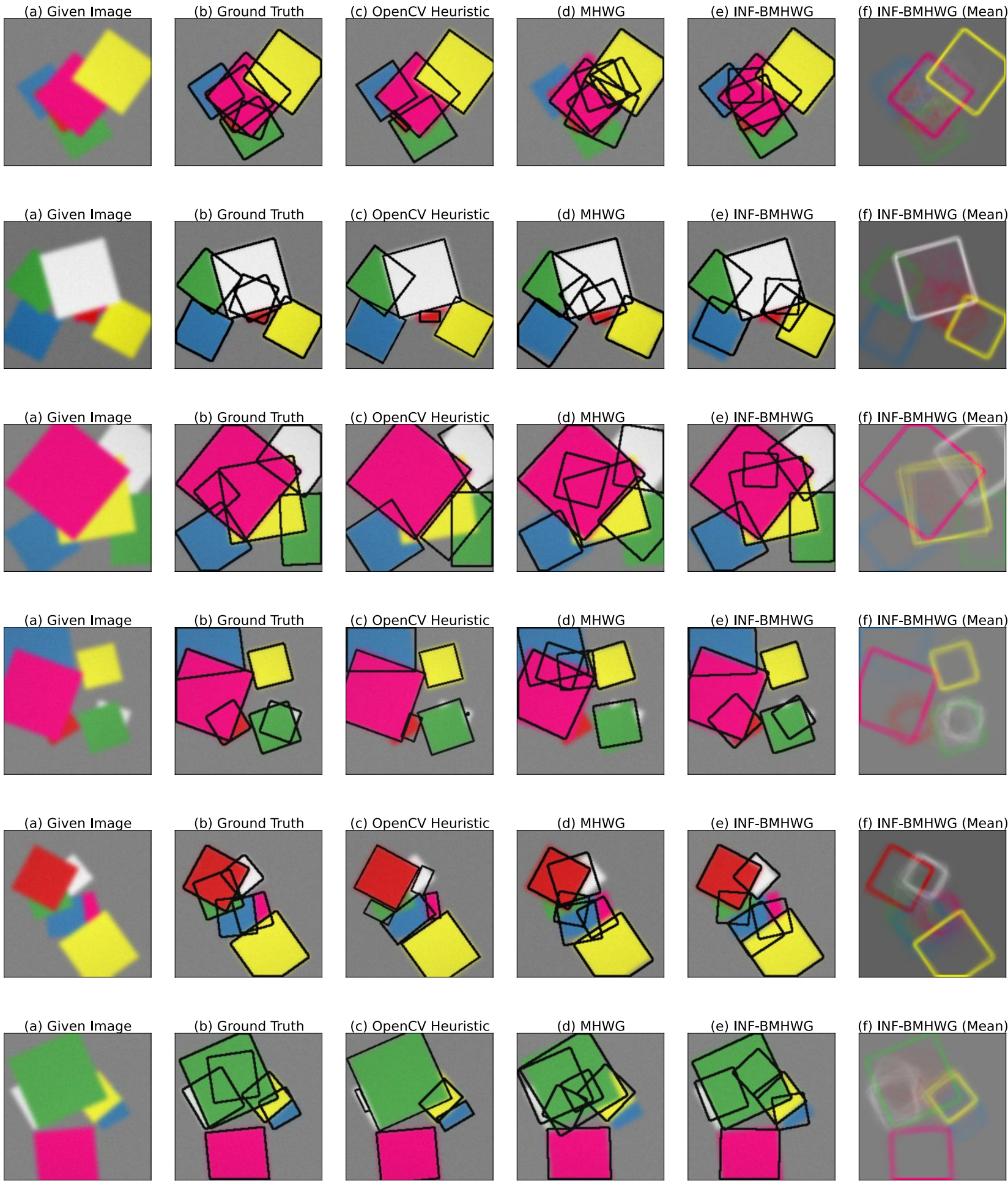
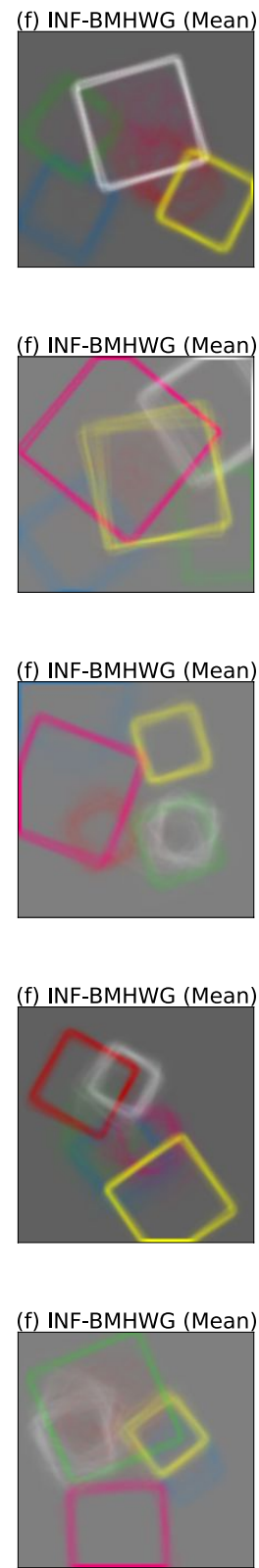

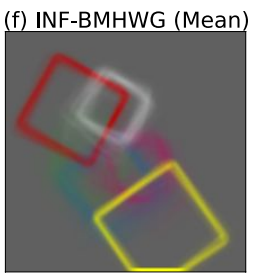

Figure 12. Qualitative Results of the occluding tiles experiment. From left to right: (a) Given image, (b) Ground truth tiles, and most probable estimates from 5000 samples obtained by (c) MHWG sampler (best baseline) and (d) our INF-BMHWG sampler. (f) Posterior expectation of the tiles boundaries obtained by INF-BMHWG sampling. (First 2000 samples are discarded as burn-in) 

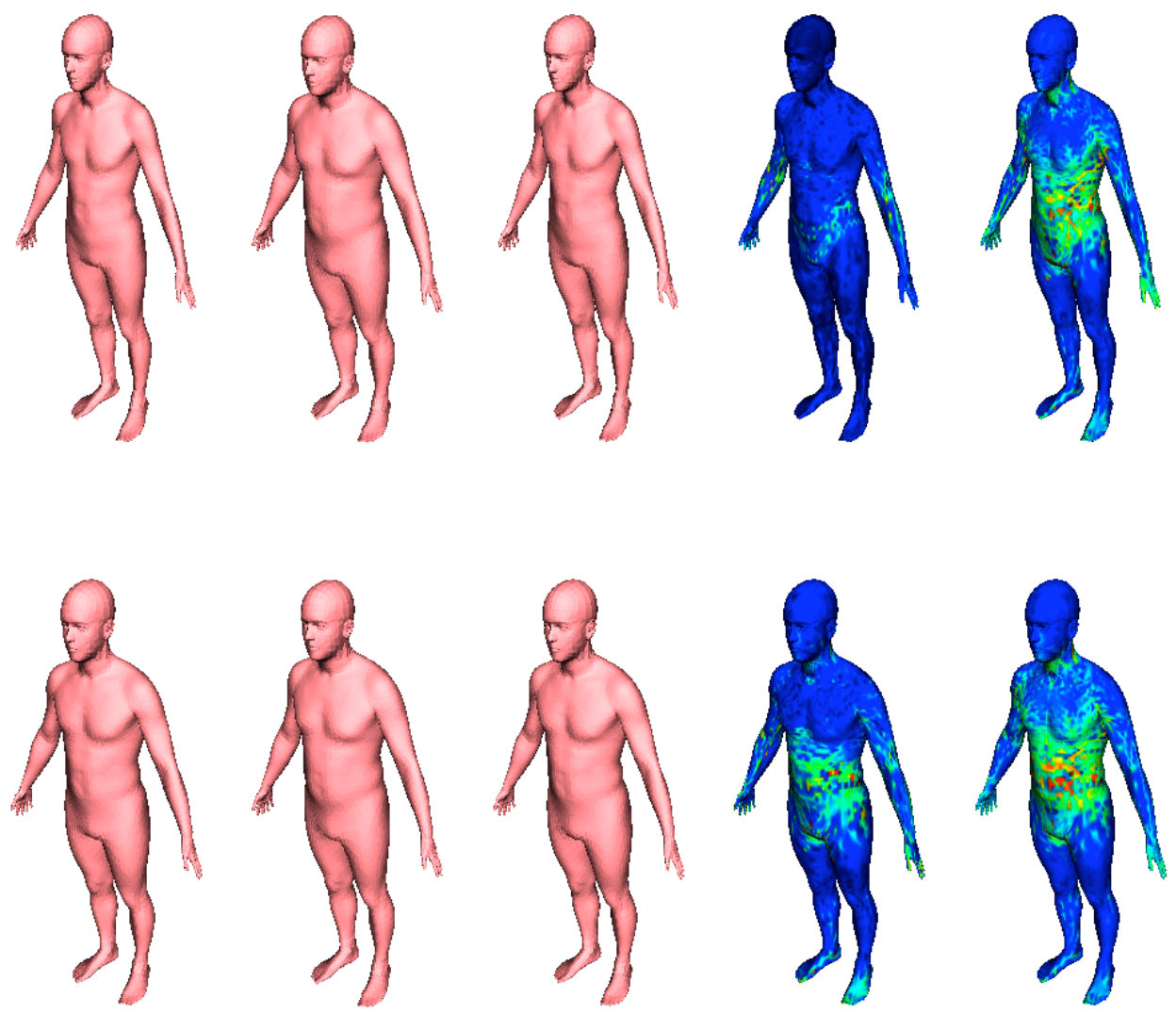

\title{
Ground Truth Mesh
}

\begin{abstract}
Mean Mesh
from Prior
\end{abstract}

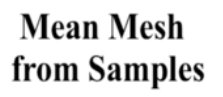

Angular
Error

Std. Deviation in
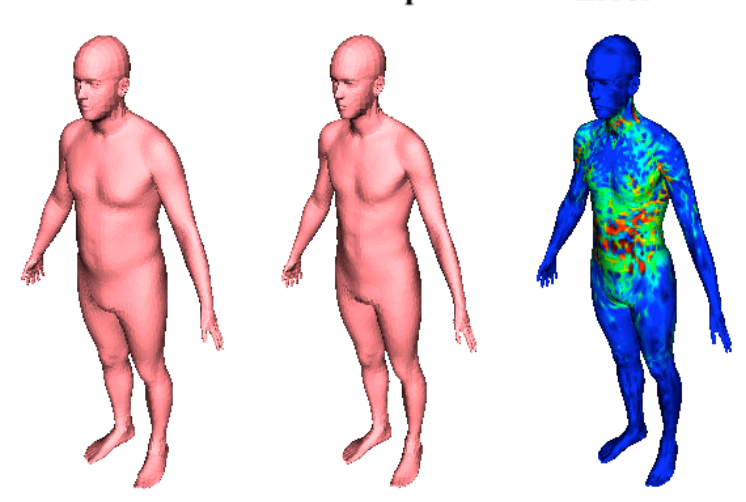

Angular Error
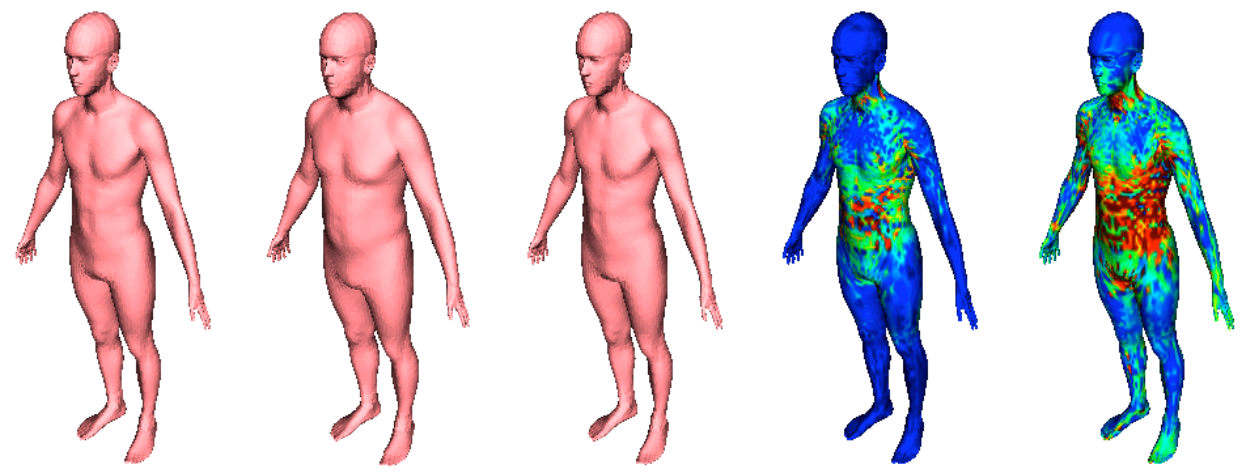

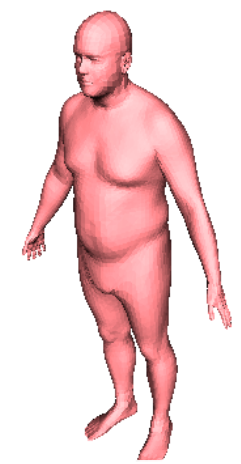

Ground Truth

Mesh

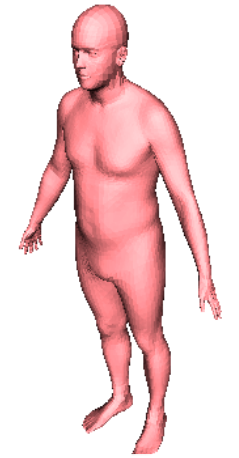

Mean Mesh from Prior

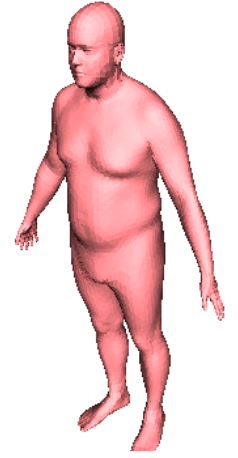

Mean Mesh from Samples

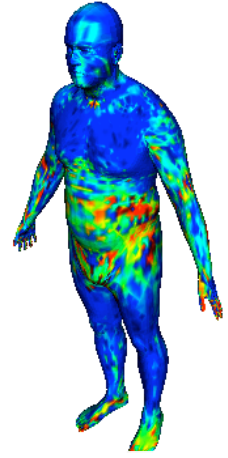

Angular Error

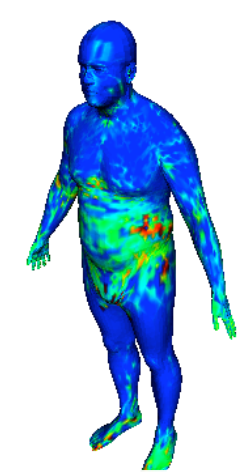

Std. Deviation in Angular Error

Figure 13. Qualitative results for the body shape experiment. Shown is the 3D mesh reconstruction results with first 1000 samples obtained using the INF-MH informed sampling method. (blue indicates small values and red indicates high values) 


\section{Supplementary Material for The Informed Sampler: A Discriminative Approach to Bayesian Inference in Generative Computer Vision Models}

\section{Varun Jampani}

VARUN.JAMPANI@TUEBINGEN.MPG.DE

Max Planck Institute for Intelligent Systems, Spemannstraße 41, 72076, Tübingen, Germany

Sebastian Nowozin

SEBASTIAN.NOWOZIN@MICROSOFT.COM

Microsoft Research Cambridge, 21 Station Road, Cambridge, CB1 2FB, United Kingdom

Matthew Loper

MLOPER@TUEBINGEN.MPG.DE

Max Planck Institute for Intelligent Systems, Spemannstraße 41, 72076, Tübingen, Germany

Peter V. Gehler

PGEHLER@TUEBINGEN.MPG.DE

Max Planck Institute for Intelligent Systems, Spemannstraße 41, 72076, Tübingen, Germany

\section{Baseline Results and Analysis}

On the next pages of this supplementary material, we give an in-depth performance analysis of the various samplers and the effect of their hyperparameters. We choose hyperparameters with the lowest PSRF value after $10 k$ iterations, for each sampler individually. If the differences between PSRF are not significantly different among multiple values, we choose the one that has the highest acceptance rate.

\section{Experiment: Estimating Camera Extrinsics}

\section{D.1. Parameter Selection}

Metropolis Hastings (MH) Figure 14(a) shows the median acceptance rates and PSRF values corresponding to various proposal standard deviations of plain $\mathrm{MH}$ sampling. Mixing gets better and the acceptance rate gets worse as the standard deviation increases. The value 0.3 is selected standard deviation for this sampler.

Metropolis Hastings Within Gibbs (MHWG) As mentioned in the main paper, the MHWG sampler with onedimensional updates did not converge for any value of proposal standard deviation. This problem has high correlation of the camera parameters and is of multi-modal nature, which this sampler has problems with.

Parallel Tempering (PT) For PT sampling, we took the best performing $\mathrm{MH}$ sampler and used different temperature chains to improve the mixing of the sampler. Figure 14(b) shows the results corresponding to different combination of temperature levels. The sampler with temperature levels of $[1,3,27]$ performed best in terms of both mixing and acceptance rate.

Effect of Mixture Coefficient in Informed Sampling (INF-MH) Figure 14(c) shows the effect of mixture coefficient $(\alpha)$ on the informed sampling INF-MH. Since there is no significant different in PSRF values for $0 \leq \alpha \leq 0.7$, we chose 0.7 due to its high acceptance rate.

\section{E. Experiment: Occluding Tiles}

\section{E.1. Parameter Selection}

Metropolis Hastings (MH) Figure 15(a) shows the results of MH sampling. Results show the poor convergence for all proposal standard deviations and rapid decrease of AR with increasing standard deviation. This is due to the high-dimensional nature of problem. We selected a standard deviation of 1.1.

Blocked Metropolis Hastings Within Gibbs (BMHWG) The results of BMHWG are shown in Figure 15(b), In this sampler we update only one block of tile variables (of dimension four) in each sampling step. Results show much better performance compared to plain MH. The optimal proposal standard deviation for this sampler is 0.7 .

Metropolis Hastings Within Gibbs (MHWG) Figure 15(c) shows the result of MHWG sampling. This sampler is better than BMHWG and converges much more quickly. Here a standard deviation of 0.9 is found to be best. 

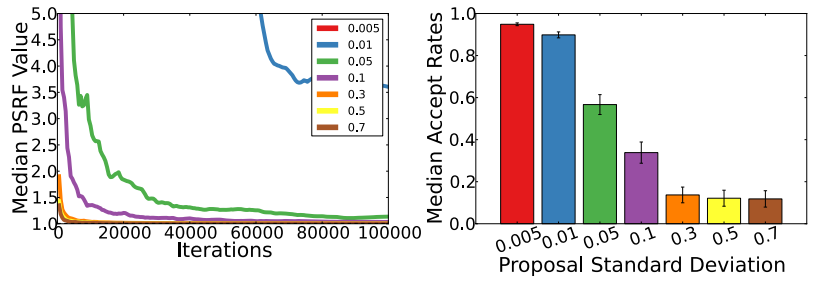

(a) $\mathrm{MH}$
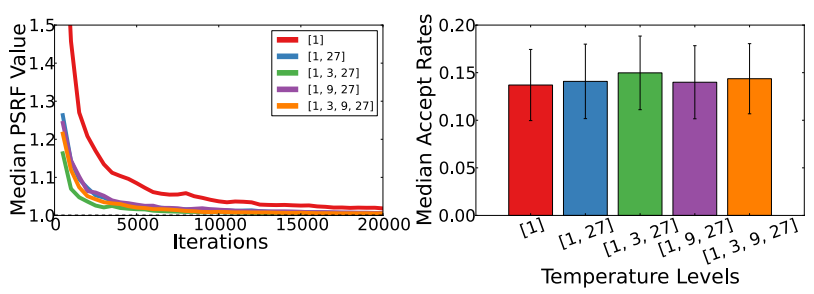

(b) PT
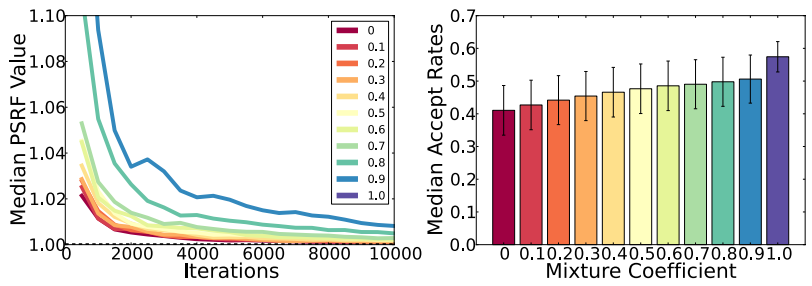

(c) INF-MH

Figure 14. Results of the 'Estimating Camera Extrinsics' experiment. PRSFs and Acceptance rates corresponding to (a) various standard deviations of $\mathrm{MH}$, (b) various temperature level combinations of PT sampling and (c) various mixture coefficients of INF-MH sampling.

Parallel Tempering (PT) The Figure 15(d) shows the results of PT sampling with various temperature combinations. Results show no improvement in AR from plain MH sampling and again $[1,3,27]$ temperature levels are found to be optimal.

Effect of Mixture Coefficient in Informed Sampling (INF-BMHWG) Figure 15(e) shows the effect of mixture coefficient $(\alpha)$ on the blocked informed sampling INFBMHWG. Since there is no significant different in PSRF values for $0 \leq \alpha \leq 0.8$, we chose 0.8 due to its high acceptance rate.

\section{F. Experiment: Estimating Body Shape}

\section{F.1. Parameter Selection}

Metropolis Hastings (MH) Figure 16(a) shows the result of $\mathrm{MH}$ sampling with various proposal standard deviations. The value of 0.1 is found to be best.

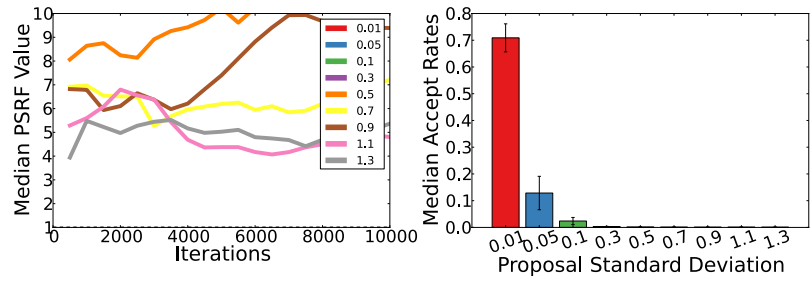

(a) $\mathrm{MH}$

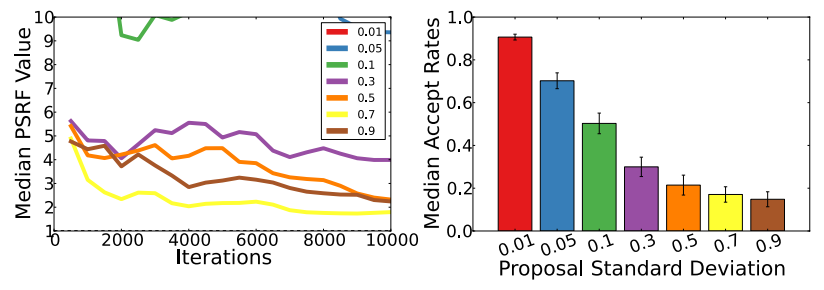

(b) BMHWG
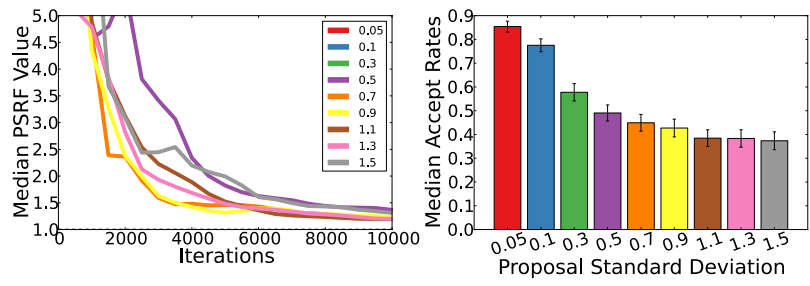

(c) MHWG
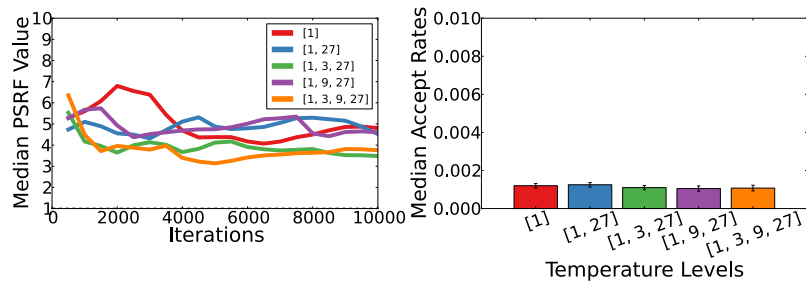

(d) PT
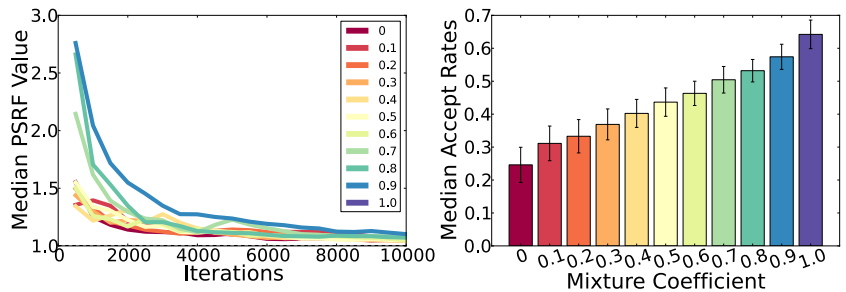

(e) INF-BMHWG

Figure 15. Results of the 'Occluding Tiles' experiment. PRSF and Acceptance rates corresponding to various standard deviations of (a) MH, (b) BMHWG, (c) MHWG,; (d) various temperature level combinations of PT sampling and; (e) various mixture coefficients of our informed INF-BMHWG sampling.

Metropolis Hastings Within Gibbs (MHWG) For MHWG sampling we select 0.3 proposal standard deviation. Results are shown in Figure 16(b) 
Parallel Tempering (PT) As before, results in Figure 16(c), the temperature levels were selected to be $[1,3,27]$ due its slightly higher AR.

Effect of Mixture Coefficient in Informed Sampling (INF-MH) Figure 16(d) shows the effect of $\alpha$ on PSRF and AR. Since there is no significant differences in PSRF values for $0 \leq \alpha \leq 0.8$, we choose 0.8 .
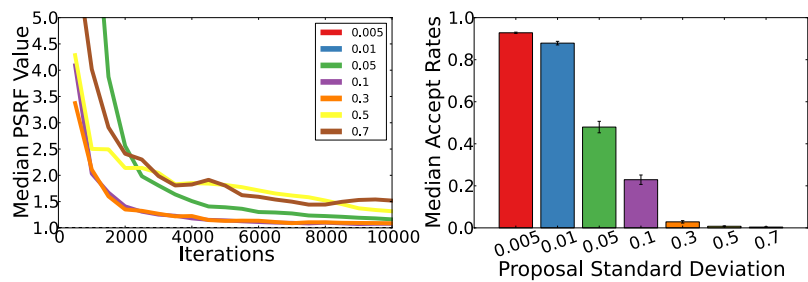

(a) $\mathrm{MH}$
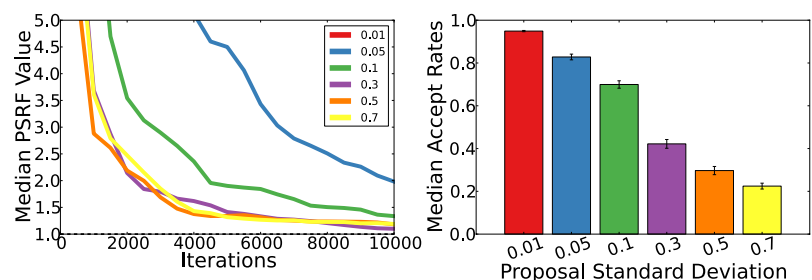

(b) MHWG
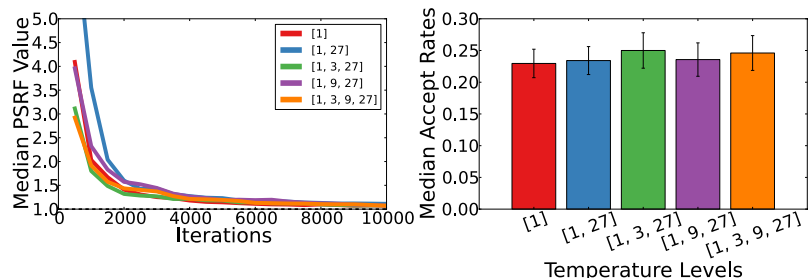

(c) PT
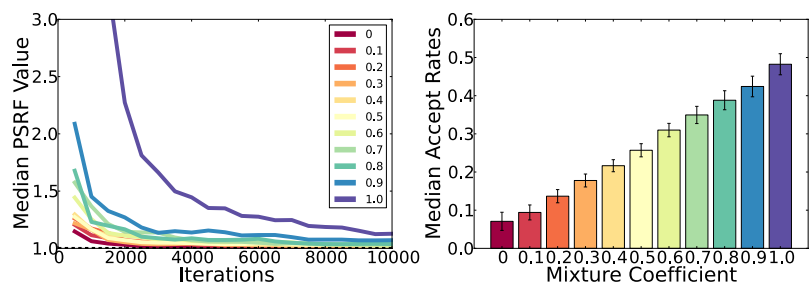

(d) INF-MH

Figure 16. Results of the 'Body Shape Estimation' experiment. PRSFs and Acceptance rates corresponding to various standard deviations of (a) MH, (b) MHWG; (c) various temperature level combinations of PT sampling and; (d) various mixture coefficients of the informed INF-MH sampling. 


\section{G. Results Overview}
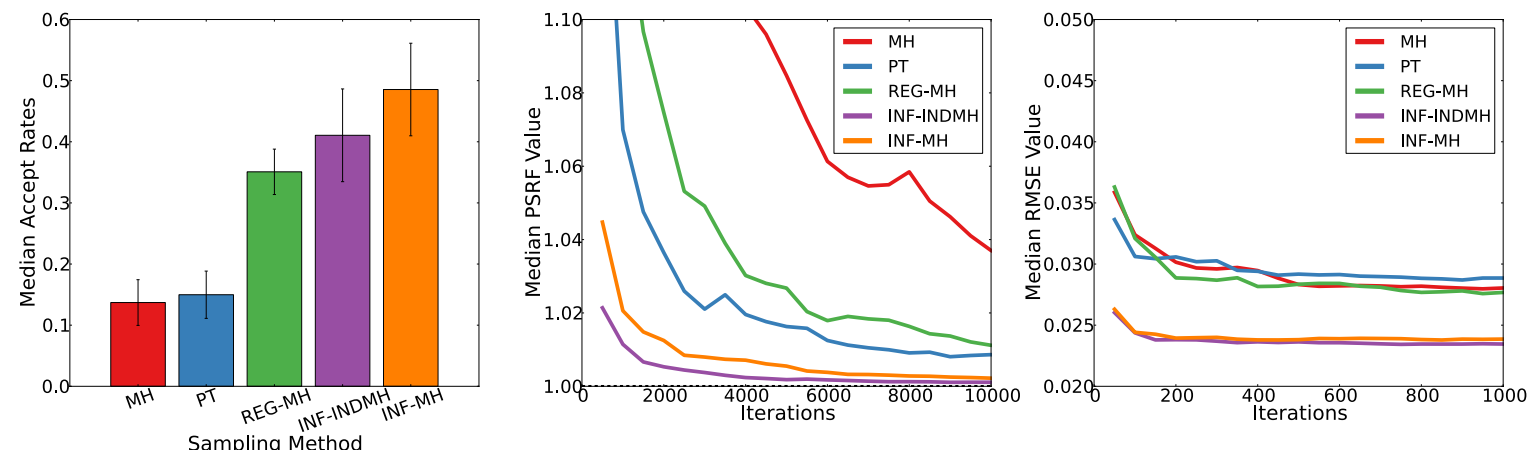

(a) Results for: Estimating Camera Extrinsics
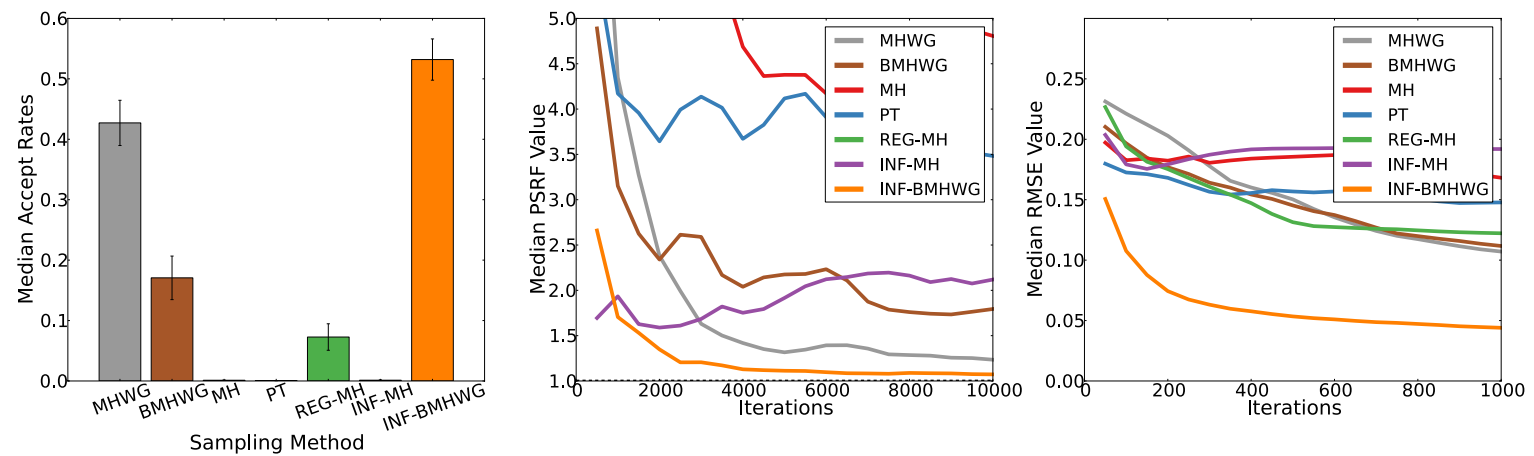

(b) Results for: Occluding Tiles
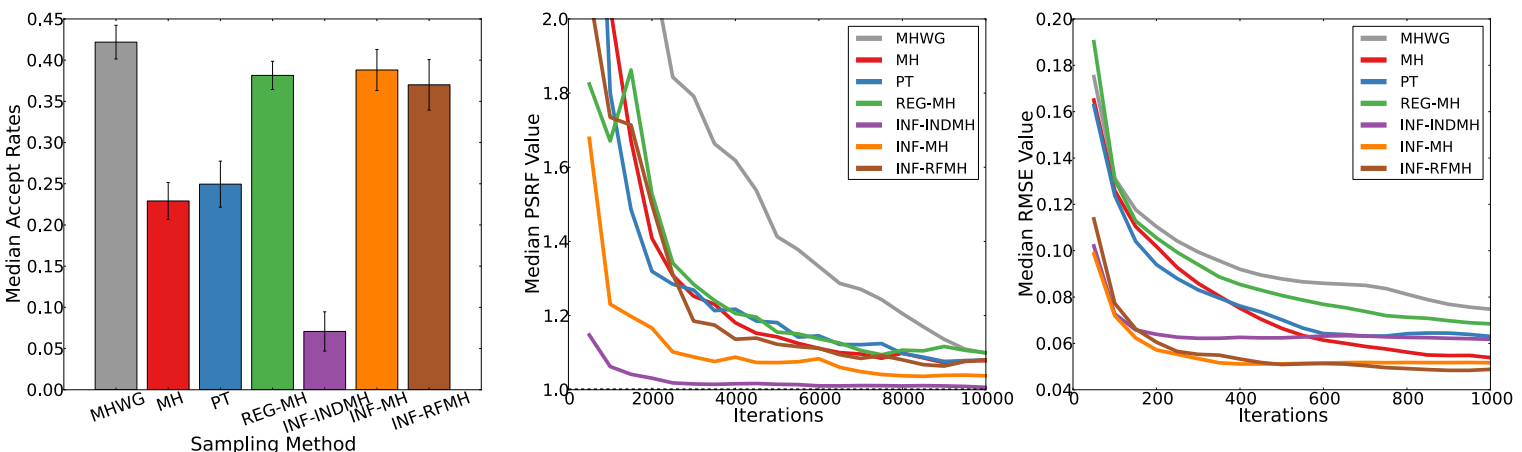

(c) Results for: Estimating Body Shape

Figure 17. Summary of the statistics for the three experiments. Shown are for several baseline methods and the informed samplers the acceptance rates (left), PSRFs (middle), and RMSE values (right). All results are median results over multiple test examples. 